\title{
REASSOCIATION PATTERNS AMONG SEGMENTAL INTERCHANGES IN MAIZE
}

\author{
D. B. WALDEN and R. C. JANCEY \\ Department of Plant Sciences, University of Western Ontario, London, \\ Ontario N6A $3 K 7$
}

Received 2.iv.75

\begin{abstract}
SUMMARY
Data from Longley (1961) and Burnham (1969) on the cytological position of the break-points in chromosomal rearrangements in maize were re-expressed in terms of segment lengths and analysed. After correction for the deviation of the distribution of break-points from an equidistribution, it was shown that highly significant excesses and deficits occurred among some but not all segment length classes. It was concluded that the pattern of reassociation shows: (i) that reassociation involves non-homologues whose interstitial segments are of similar length more frequently than expected; (ii) that the two interchange segments involved in a translocation are of similar length more frequently than expected.
\end{abstract}

\section{INTRODUCTION}

AN extensive literature exists on the genetic effects and consequences of exposure to radiation and radiomimetic and radiochemical compounds. The origin of chromosomal aberrations had long been considered to be the consequence of "breakage and reunion" until Revell (1955) proposed an "exchange" hypothesis as an alternative. The distribution of the "breakage" or " exchange" events has been approximately determined in several species, with a " random " distribution being claimed by some workers and a "non-random" distribution favoured by several recent investigators (e.g. Caspersson et al., 1972). It is important for the present communication to note that most of the studies record observations made one or a few cell generations after exposure to the mutagen and that only limited information is available about the survival of the aberrations so induced.

For at least two species, Drosophila melanogaster and Zea mays, the data are extensive enough to justify analysis (Jancey and Walden, 1972). The maize data are drawn from more than 1000 translocations and are based on measurements made by Longley (1961) and corrections provided by Burnham (1969). The data include almost all of the translocations known to have survived, with the exception of Morris's (1954a, $b, 1955)$ stocks.

The question of selection, during the period from induction (or discovery) until analysis, of certain types of aberrations cannot be assessed other than to point out that: (i) Longley (1961) and others required each aberration to be passed through at least one reproductive cycle before analysis; and (ii) limited data (Lin, 1972) suggest that mitomycin C and hydroxylamine sulfate produce aberration profiles in maize similar to those described in the present paper. The population of aberrations under analysis therefore represents a sample of those compatible with survival. 
The population was described earlier (Burnham, 1962; Jancey and Walden, 1972) as one in which the break-points were not distributed equally along the chromosome arms of maize. It will be demonstrated in this communication that the pattern of reassociation between arms of nonhomologues differs from an equidistribution to an extent exceeding that attributable to the previous inequality.
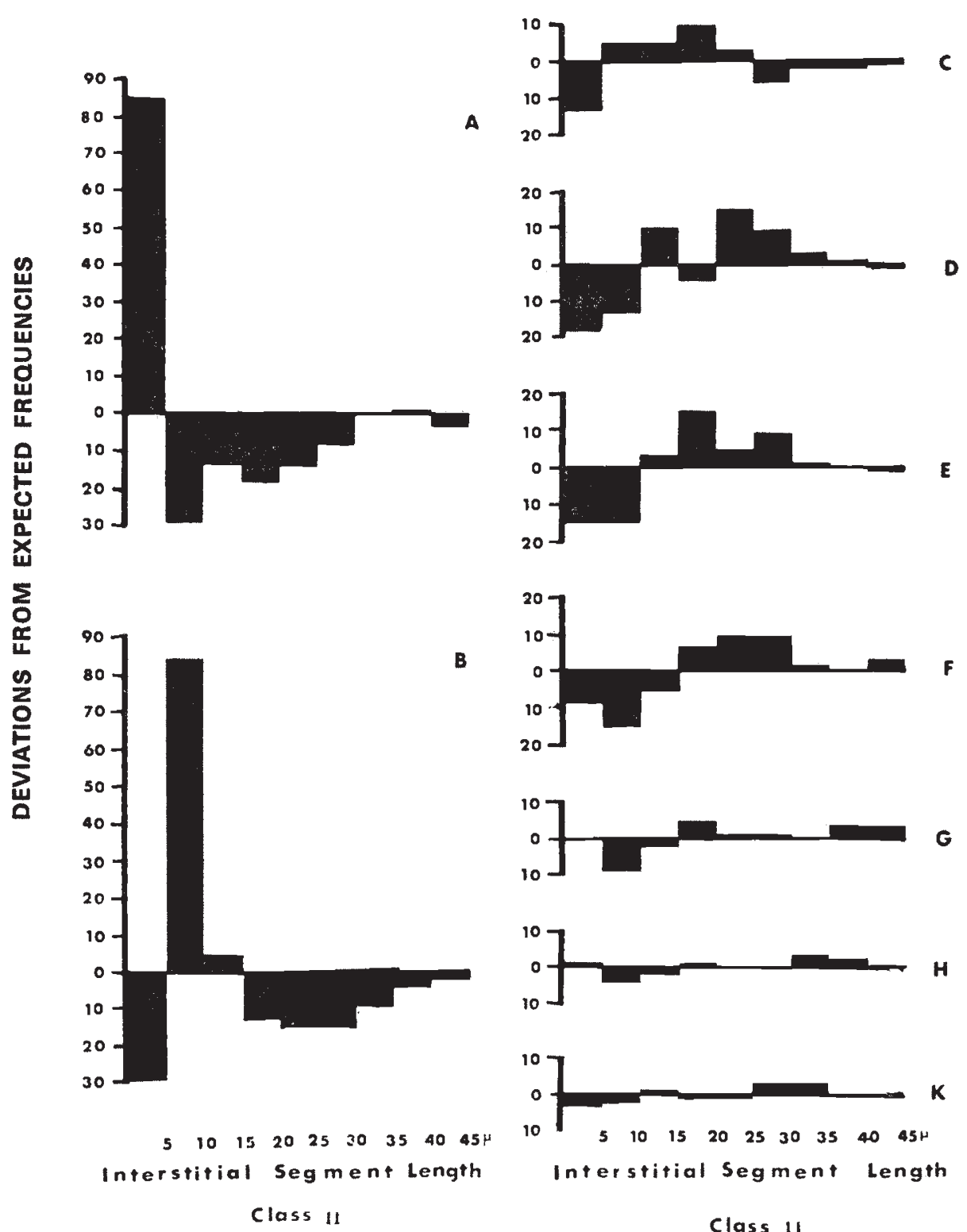

Class 11

Fig. 1.-Deviations from expected frequency of reassociation events: interstitial segment (class I) vs interstitial segment (class II). For class assignment procedure, see Materials and Methods. Histogram A, interstitial segment (class I) includes segments of length from centromere $-4.9 \mu$; histogram B, segments of length $5 \cdot 0-9 \cdot 9 \mu$ from centromere, etc. 

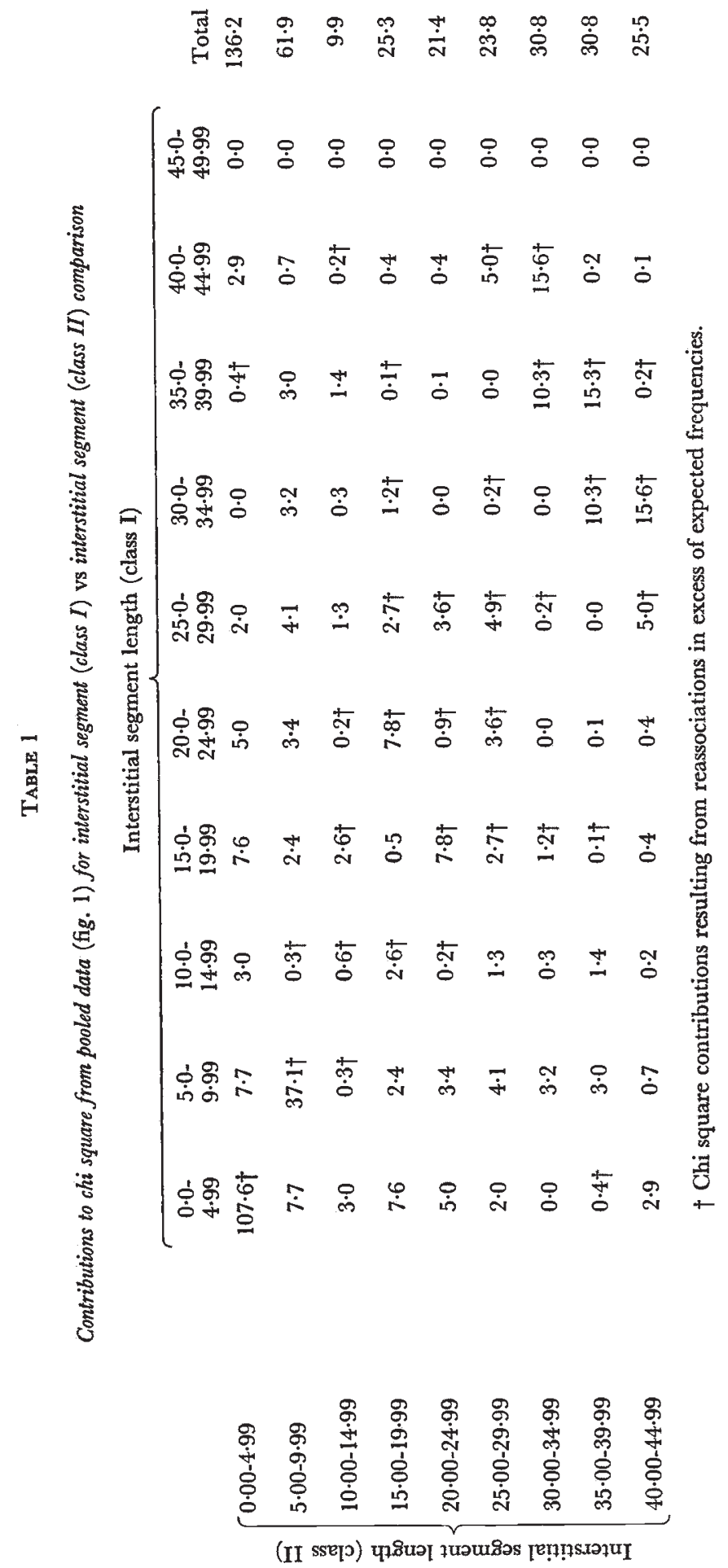


\section{Materials and Methods}

The null hypothesis was erected that the reassociation between chromosome segments, pooled for long and short arms, was at random, taking into account the correction for the distribution of break-points described earlier (Jancey and Walden, 1972). Data from Longley (1961) and Burnham (1969) were expressed in micron units as measured at pachynema and on this basis the chromosomes were divided into $5 \mu$ divisions. If the pooled data are divided into $n$ frequency classes and if $f=$ the observed break-point frequency per class, then the expected frequency of reassociation $R$, for the $i$ th row and $j$ th column intersection in the $n \times n$ symmetric matrix of reassociation frequencies will be:

$$
R_{i j}=\sum_{i=1}^{n} f_{i j}\left(\sum_{j=1}^{n} f_{i j} / \sum_{i=1}^{n} \sum_{j=1}^{n} f_{i j}\right) .
$$

From this, a test of significance of departures from expectation can readily be obtained using chi square values.

The descriptions of the translocations follow the system of Longley (1961). The chromosome segment between the centromere and the break-point is termed the interstitial and that distal to the break-point the interchange segment. Class I in figs. 1, 2 and 3 is always the first-mentioned chromosome of the pedigree, e.g. $\mathrm{T}_{8-6} \mathrm{~b}=8 \mathrm{~S} .76,6 \mathrm{~L} .79(\mathrm{~S}=$ short arm; $\mathrm{L}=$ long arm; for $8 \mathrm{~S}$, interstitial segment $=0.76$ of the arm length, interchange segment $=0.24$ of the arm length; for $6 \mathrm{~L}$, interstitial segment $=0.79$ of the arm length, interchange segment $=0.21$ of the arm length; in fig. 1 and table 1 , interstitial class I contains the segment $8 \mathrm{~S}=0.76$ and class II the segment $6 \mathrm{~L}=0 \cdot 79$ ).

\section{REsults}

The frequencies of reassociation are presented in figs. 1, 2 and 3 in the form of deviations from expected values. In fig. 1 the nine histograms correspond to class intervals along the length of the chromosome arms. Thus, in histogram A, sites occurring between $0.0 \mu$ and $4.9 \mu$ from the centromere show an excess of 85 reassociations in which the interstitial segment of the non-homologue (class II) was a segment also of 0.0-4.9 $\mu$ in length. In histogram $A$ it will be seen also that there was a deficit of 30 reassociations in which the relict interstitial segment was 5.0-9.9 $\mu$ in length. Subsequent histograms comprising fig. 1 show similar excesses of reassociation where interstitial segment lengths of class II are identical to those of class I, and mainly deficits where they are of different lengths. Interstitial segments of class I show this somewhat less clearly as distances from the centromere increase and the size of deviations decrease. Both features may be attributed to the lower frequency of break-points in regions distant from the centromere plus the fact that arms are of different length.

In fig. 2, the comparisons of interstitial segment classes and interchange segment classes are plotted. In fig. 2, histogram A, it will be seen that the interstitial segment class $0.0-4.9 \mu$ from the centromere shows an excess of reassociations with the longer interchange segment classes, and a deficit with the shorter ones. The distinction is less marked than that seen in histogram A of fig. 1. This may be attributed to the differing total length of chromosome 
arms, which, when classified into an interstitial segment class with length $4.9 \mu$ from the centromere, will give rise to a variety of interchange segment lengths.
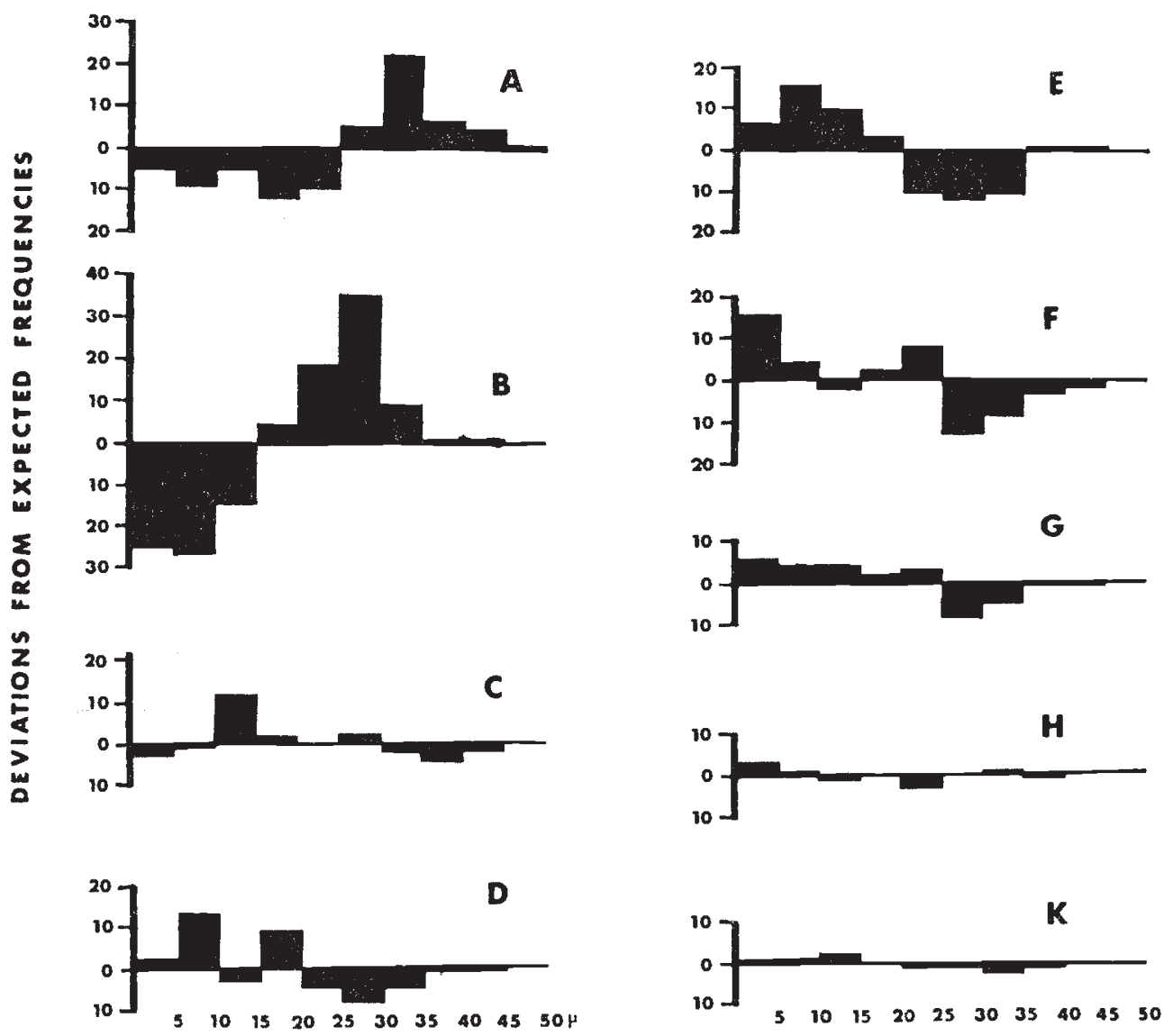

\section{Interchange Segment length Class 11}

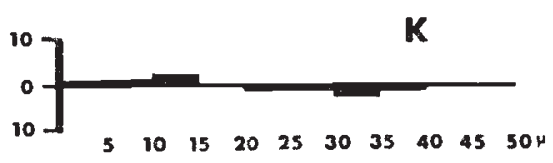

\section{Interchange Segment length \\ Class II}

FIG. 2.-Deviations from expected frequency of reassociation events; interstitial segment (class I) vs interchange segment (class II). For class assignment procedure, see Materials and Methods section. Histogram A, interstitial segment (class I) includes segments of length from centromere- $4.9 \mu$; histogram $B$, segments of length 5.0-9.9 $\mu$ from centromere, etc.

In fig. 3 a consistent pattern emerges from the comparison of interchange $v s$ interchange segment length: an excess of reassociation has occurred in which the interchange segments are of similar length while there is usually a deficit of reassociation in which the interchange segment lengths were markedly dissimilar. It should be noted that in fig. 3 one is " reading" the arm from the telomere rather than from the centromere, i.e. histogram A demonstrates that there is an excess of reassociations in which the interchange segments are both short. 

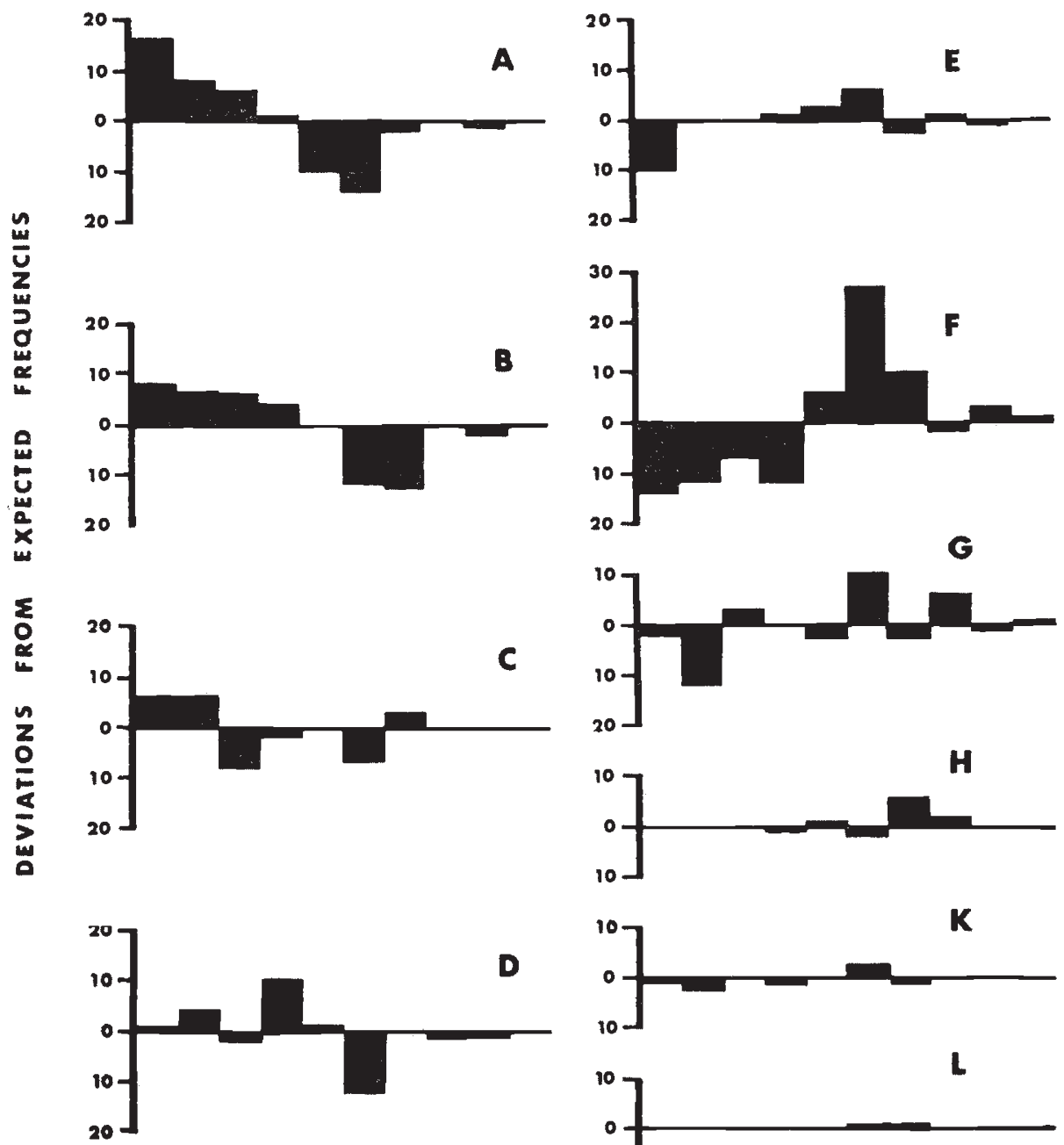

$\begin{array}{llllllllll}5 & 10 & 15 & 20 & 25 & 30 & 35 & 40 & 45 & 50 \mu\end{array}$
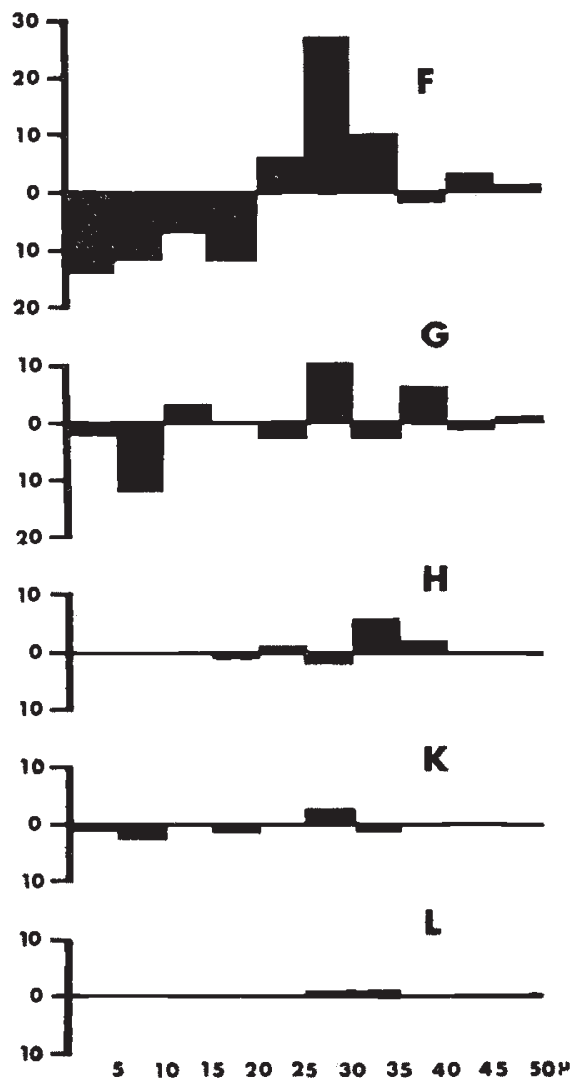

Interchange Segment Length

\section{Class II}

Class II

FIG. 3.-Deviations from expected frequency of reassociation events: interchange segment (class I) vs interchange segment (class II). For class assignment procedure, see Materials and Methods section. Histogram A, interchange segment (class I) includes segments of length $0 \cdot 0-4 \cdot 9 \mu$; histogram $B$, segments of length $5 \cdot 0-9 \cdot 9 \mu$, etc.

Contributions to $\chi^{2}$ values are listed in tables 1,2 and 3 . These values reflect the pattern seen in the histograms, though the relatively low expected values associated with sites distant from the centromere tend to increase the $\chi^{2}$ contributions from those regions. Results expressed wholly in terms of interstitial lengths are highly significant, both as regards individual segment classes and in total. The only exception to this was for interstitial segment class $I$ in the region $10 \cdot 0-14.9 \mu$. It will be recalled from fig. 1 that the 

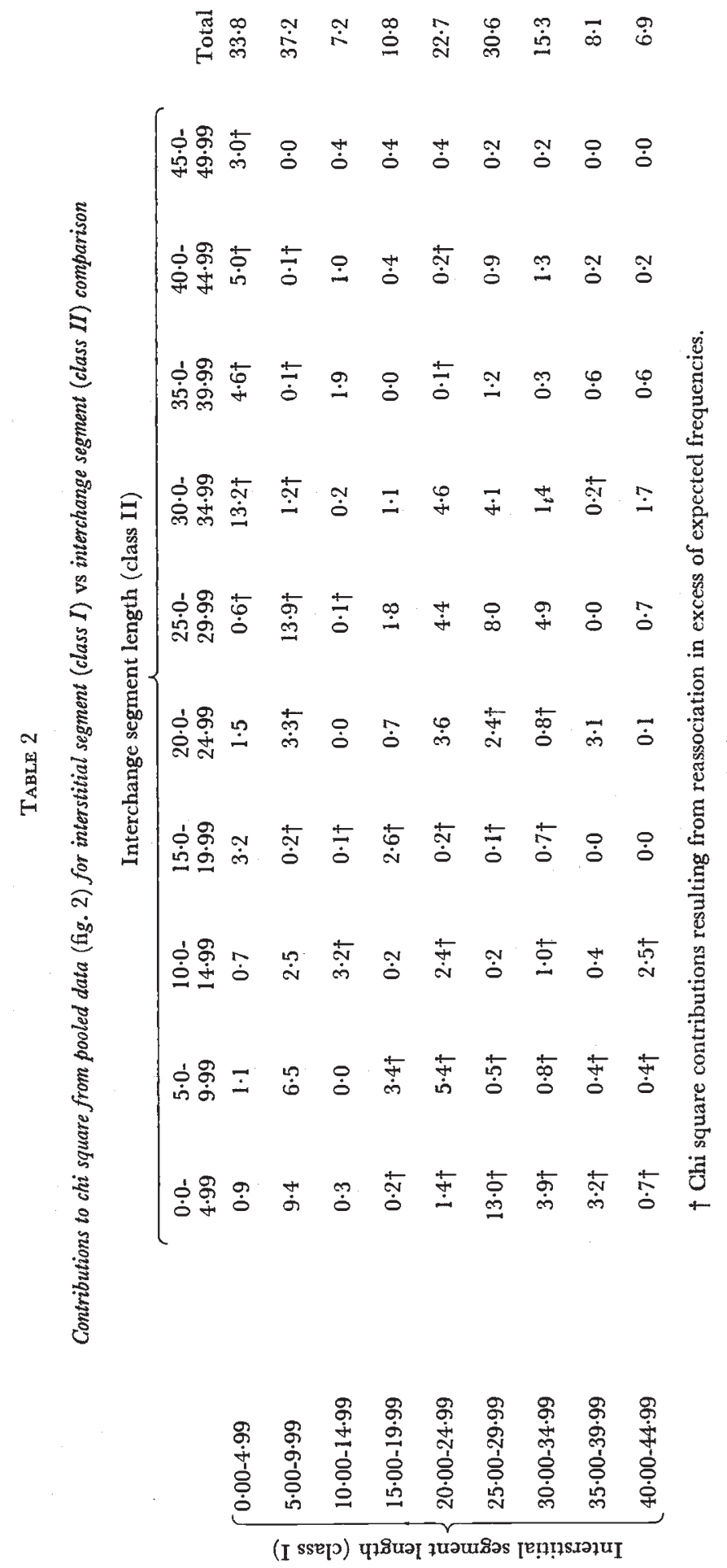

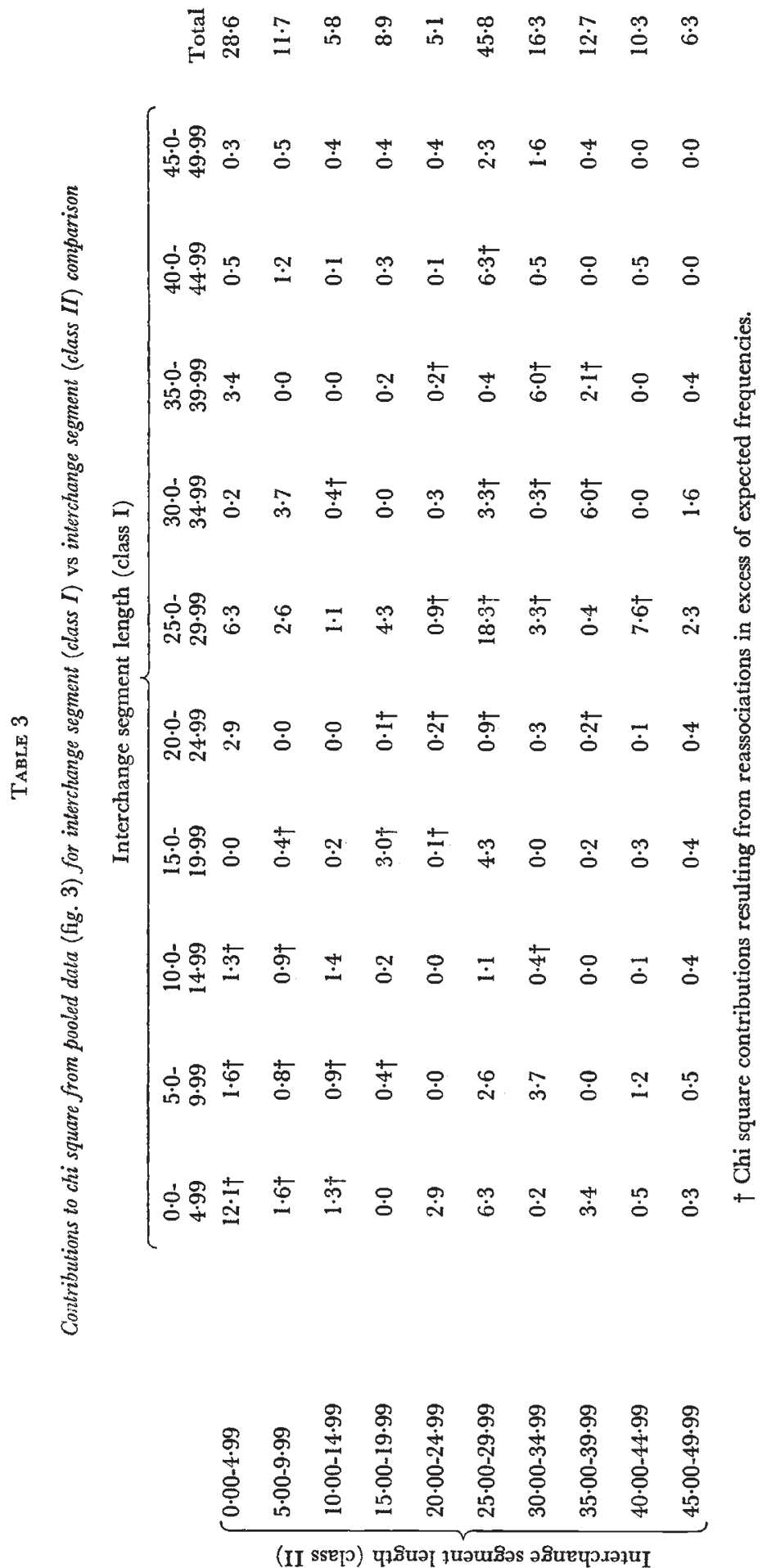
pattern tended to be somewhat less obvious for interstitial segments falling in about the mid-range of chromosome arm lengths. Tables 2 and 3 , while still showing some significant values, parallel the second and third figures in their progressive obscuring of the pattern seen in fig. 1 and table 1 . The telomere related events are however apparent in table 3 with significant values running on the upper left to lower right diagonal.

\section{Discussion}

The data used in this study are necessarily composed of records of those events which proved to be compatible with survival. There seems to be no a priori reason to suppose that such a filtering would produce the pattern of deviations shown in this study, though such a possibility cannot be wholly discounted.

\section{(i) Ascertainment and specific distributions}

There have been two large populations of translocations studied in maize. Morris (1954a, b, 1955, and in Koo, 1959) has shown that of 600 translocations, 18 involved arms of homologous chromosomes, providing a very close approximation of Koo's expectation based on a 36:1 ratio for non-homologues: homologues for reciprocal translocations. Morris's translocations were induced in dry, dormant seed and analysed in diakinesis. There are no translocations involving homologous chromosomes among the 952 translocations analysed in the present study (Longley, 1961).

Evans (1962) has reviewed the early literature in which there are claims for a random distribution of aberrations and an almost equal number of demonstrations of a non-random distribution. Recent reports establish that the distribution of induced aberrations is a function of the agent, the culture conditions, the phase of the nuclear cycle, the species and the precise cytogenetic composition of the complement. It is also possible that confusion has arisen because there are two kinds of " distribution " about which statements as to randomness may be made. There is (1) the distribution of break-points among chromosome regions and (2) the pattern of reassociation of these break-points; one may be random without the other being so.

There is thus no necessary conflict between the claim of Koo (1959, using data from Morris $(1954 a, b, 1955)$ for the random occurrence of translocation in maize and reports of significant deviations from an equidistribution. Likewise, in Tradescantia, Watanabe (1973) reports that the translocations occurred at random whereas Sax and Mather (1939) and Swanson (1942) have shown that the events are not equally distributed along the chromosome.

\section{(ii) Interstitial and interchange segments}

Correction for the frequency of reassociation has permitted the demonstration that the pattern of reassociation itself deviates from an equidistribution. It is shown from the analysis above that in maize the most important feature in the siting of reassociation is the distance of the site from the centromere. Interestingly, this conclusion was reached on other grounds by Hinton (1965) from a study of X chromosome aberrations in D. melanogaster and Vicia by Rieger et al. (1973) and can be inferred from Kaufmann (1946) from his analysis of inversions and translocations in $D$. melanogaster. 
Along the entire length of a chromosome arm an excess of reassociation occurs with non-homologues in which the second break-point produces an interstitial segment of similar length (fig. 1, table l). This is exceptionally clear in histograms A and B, fig. l. The identical conclusion is inferred from an examination of fig. 2 in which the short interstitial classes show a deficit of expected reassociation with short interchange segments but an excess with the longer interchange segments. Similarly, the longer interchange segments (fig. 2, histograms E, F and G) show an excess of reassociation with the short interstitial segments and a deficit with the longer ones.

The pattern is less obvious in fig. 3, although in general a short interchange segment is more likely to be involved in a translocation with a second short interchange segment than a longer one (fig. 3, histograms, A, B and C). A long interchange segment is more likely to be involved with a second long one (fig. 3, histograms E, F and G) than a shorter one contrary to the observation (Burnham, 1962) that indicated there was ". . . no strong tendency for species of equal length to be interchanged".

Of the 51 translocations involving a centromere that were not included in the present analysis, we reported (Jancey and Walden, 1971) that 43 of 51 involved two centromeres. This frequency was a highly significant deviation from an equidistribution and is similar to that reported in some other species (e.g. Caspersson et al., 1972).

Longley (1961), and as summarised in Burnham (1962), concluded that in maize there appeared to be some preferential involvement of regions of heterochromatin in translocation sites. Evans and Bigger (1961) and several others have reported a similar finding and have proposed that this preferential involvement may be related to other characteristics of heterochromatin, such as association to non-homologous regions or attachment to the nuclear membrane.

Thus, it appears that there are sufficient reports of species (Grant, 1965) and cytogenetic differences (e.g. Kaufmann, 1946; Michaelis and Rieger, 1968; Morad et al., 1973) to discount most generalisations which attempt to comment on specific units of a chromosome. Nevertheless, the question remains as to whether or not there exists a pattern or organisation of a nucleus which may, once understood, reveal an explanation for seemingly contradictory observations.

\section{(iii) Spatial arrangement of the nucleus}

Independent observations from a number of laboratories, based on a variety of technologies and species, continue to point to the view that at all times in the nuclear cycle there is a multi-dimensional organisation of the nucleus. Radiobiologists have considered the possible influence of spatial arrangement (Wolff et al., 1958) on the occurrence (frequency and type) of aberration. Sax (1940) was particularly forthright: ". . . an analysis of the loci of breaks involved in fusions between different chromosomes or chromosome arms should indicate the arrangement and relations of the chromosomes at various phases in the nuclear cycle". It would seem now that the independent observations by the early cytologists (particularly Rabl and Boveri) which led to the concepts of the polarity and stationary nature of the chromosome during interphase (Wilson, 1925) and to which attention has been called periodically (Grell, 1969) take on an added importance as reports 
become available from several disciplines which leave no doubt that there is an organisation and probably a topographical pattern to the interphase nucleus. That the former is part of the genotype and the latter yet another phenotype now seems obvious. It is not unexpected that species and other differences may exist at the genotypic level.

Our conclusions from the maize translocations are compatible with a model containing the features proposed by Evans (1961) in which the centromeres are attached to a limited area of the nuclear membrane, thereby accounting for the unexpectedly high centromere-centromere exchanges, with the telomere regions likely attached to the membrane at some point remote from the centromeres and with a greater mutual spatial separation between them than in the case of the centromeres. Jancey (1975) has been able to show on a purely statistical basis that the centromeres and adjoining $5 \mu$ segment classes from the maize data are markedly grouped in a Principal Components Analysis, quite reminiscent in illustrative fashion of a polarised nucleus.

The excess of reassociations involving centromeres only and the excess involving interstitial segments of similar length lends itself to the simulation of a nucleus in which the arms radiate from the centromere attachment area in an extended, near linear, manner rather than in a highly intertwined matrix. In such a model, overlappings, particularly in the intercalary regions and occasional intercalary attachment sites (heterochromatic knobs?) to the nuclear membrane, would be expected. Such an arrangement is similar to that conceived necessary to account for the affinity distances of somatic metaphase chromosomes described by Horn and Walden (1976).

Acknowledgments.-A major portion of this manuscript was prepared while the senior author was Visiting Professor, Department of Genetics, University of Birmingham. He wishes to thank Professor J. L. Jinks for the opportunity to be in the Department and several members of the Department for earnest and stimulating discussion. This research was supported by a National Research Council of Canada Grant.

\section{REFERENCES}

burnham, c. R. 1962. Discussions in Cytogenetics. Burgess Publishing Co., Minneapolis. BURNHAM, c. R. 1969. In IV. Report on maize cooperative by R. J. Lambert. Maize Genet. Coop. News Letter, 43, 230.

CASPERSSON, T., HAGLUND, U., LINDELl, B., AND ZECH, L. 1972. Radiation-induced nonrandom chromosome breakage. Exptl Cell Res., 75, 541-543.

Evans, H. J. 1961. Chromatid aberrations induced by gamma irradiation. I. The structure and frequency of chromatid interchanges in diploid and tetraploid cells of Vicia faba. Genetics, 46, 257-275.

EVANS, H. J. 1962. Chromosome aberrations induced by ionizing radiations. Int. Rev. Cytol., 13, 221-321.

EVANS, H. J., AND BIGGer, T. R. L. 1961. Chromatid aberrations induced by gamma irradiation. II. Non-randomness in the distribution of chromatid aberrations in relation to chromosome length in Vicia faba root-tip cells. Genetics, 46, 277-289.

GRANT, c. J. 1965. Chromosome aberrations and the mitotic cycle in Trillium root tips after X-irradiation. Mut. Res., 2, 247-262.

Grell, R. F. 1969. In: Genetic Organization-A Comprehensive Treatise. Ed. by Caspari, E. W., and Ravin, A. Vol. 1. Academic Press, New York.

HINTON, c. w. 1965. The effects of heterozygous autosomal translocations on recombination in the X chromosome of $D$. melanogaster. Genetics, 51, 971-982.

HORN, J. D., AND WALDEN, D. B. 1976. Affinity distance values among somatic metaphase chromosomes in maize. Genetics (in press). 
JANGeY, R. C. 1975. Evidence for the polarized nucleus in maize. Canad. F. Genet. Cytol., 17, 245-252.

JANCEY, R. C., AND WALDEN, D. B. 1971. Reanalysis of Longley's data: translocations in maize. Maize Genet. Coop. Newes Letter, 45, 195-203.

JANGEY, R. C., AND WALDEN, D. B. 1972. Analysis of pattern in distribution of breakage points in the chromosomes of Zea mays L. and D. melanogaster Meigen. Canad. 7. Genet. Cytol., 14, 429-442.

KAUfMANN, B. P. 1946. Organization of the chromosome. I. Break distribution and chromosome recombination in Drosophila melanogaster. F. Exptl Zool., 102, 293-320.

Koo, F. K. s. 1959. Expectations on random occurrence of structural interchanges between homologous and between non-homologous chromosomes. Amer. Nat., 93, 193-199.

LIN, M. s. 1972. Chromosomal aberrations in relation to the nuclear cycle in Zea mays L. $\mathrm{Ph} . \mathrm{D}$. Thesis, University of Western Ontario, London, Ontario.

LONGLEY, A. E. 1961. Breakage points for four corn translocation series and other corn chromosome aberrations. Crop. Res., U.S. Dept. Agr., Agr. Res. Service, ARS 34-16.

MICHAELIS, A., AND RIEGER, R. 1968. On the distribution between chromosomes of chemically induced chromatid aberrations: Studies with a new karotype of Vicia faba. Mut. Res., $6,81-92$.

morad, M., Jonasson, J., AND Lindston, J. 1973. Distribution of Mitomycin c induced breaks on human chromosomes. Hereditas, 74, 273-282.

MORRIs, R. 1954a. Effects of irradiating dormant maize seeds on translocation frequencies at meiosis. Maize Genet. Coop. Newes Letter, 28, 66-67.

MoRRIs, R. 1954b. Translocations between homologous chromosomes in maize. Maize Genet. Coop. Newes Letter, 28,68

MORRIS, R. 1955. Induced reciprocal translocations involving homologous chromosomes in maize. Am. F. Bot., 42, 546-550.

REVELL, s. H. 1955. A new hypothesis for chromatid changes. In: Proc. of the Radiobiology Symp., Liege, 1954, 243-253. Butterworths, London.

RIEGER, R., MICHAELIS, A., SCHUBERT, I., AND MEISTER, A. 1973. Somatic interphase pairing of Vicia chromosomes as inferred from the hom/het ratio of induced chromatid interchanges. Mut. Res., 20, 295-298.

sAx, K. 1940. An analysis of X-ray induced chromosomal aberrations in Tradescantia. Genetics, 25, 41-68.

SAX, K., AND MATHER, K. 1939. An X-ray analysis of progressive chromosome splitting. 7. Genet., 37, 483-490.

swanson, c. P. 1942. The effects of ultra-violet and x-ray treatment on the pollen tube chromosomes of Tradescantia. Genetics, 27, 491-503.

WATANABE, H. 1973. Cytogenetic studies of reciprocal translocations induced by $\mathrm{x}$-rays in Tradescantia paludosa. Heredity, 31, 85-94.

wilson, E. B. 1925. The Cell in Development and Heredity. Third edition. Macmillan Co., New York.

WOLFF, S., ATWOOD, K. C., RANDOLPH, M. L., AND LUIPPOLD, H. E. 1958. Factors limiting the number of radiation-induced chromosome exchanges. I. Distance: evidence from non-interaction of $\mathrm{x}$-ray and neutron-induced breaks. 7. Biophys. Biochem. Cytol., 4, $365-372$. 